\title{
Underwater Communications and Networking
}

\author{
Charalampos C. Tsimenidis, ${ }^{1}$ Yuriy Zakharov, ${ }^{2}$ Christophe Laot, ${ }^{3}$ \\ Konstantinos Pelekanakis, ${ }^{4}$ Paolo Casari, ${ }^{5}$ and Andrey K. Morozov ${ }^{6}$ \\ ${ }^{1}$ School of Electrical and Electronic Engineering, Newcastle University, Merz Court, Newcastle upon Tyne NE1 7RU, UK \\ ${ }^{2}$ Department of Electronics, University of York, Heslington, York YO10 5DD, UK \\ ${ }^{3}$ Institut Mines-Télécom, Telecom Bretagne, UMR CNRS 6285 Lab-STICC, Université Européenne de Bretagne, \\ Technopôle Brest Iroise, CS 83818, 29238 Brest Cedex, France \\ ${ }^{4}$ Acoustic Research Laboratory, National University of Singapore, 12A Kent Ridge Road, Singapore 119223 \\ ${ }^{5}$ Department of Information Engineering, University of Padova, Via G. Gradenigo, 6/B, 35131 Padova, Italy \\ ${ }^{6}$ Applied Ocean Physics and Engineering, Woods Hole Oceanographic Institution, MS No. 9, Bigelow 404, Woods Hole, \\ MA 02543, USA
}

Correspondence should be addressed to Charalampos C. Tsimenidis, charalampos.tsimenidis@ncl.ac.uk

Received 13 December 2012; Accepted 13 December 2012

Copyright ( 2012 Charalampos C. Tsimenidis et al. This is an open access article distributed under the Creative Commons Attribution License, which permits unrestricted use, distribution, and reproduction in any medium, provided the original work is properly cited.

In the last two decades, we have witnessed growing research interest in underwater communications and networks for civilian and scientific, as well as military type of applications. Despite the plethora of studies in this area, the hostile nature of the propagation medium poses many challenging issues, many of which are still to be solved. The underwater acoustic communications channel (UAC) can be characterized in most cases as a doubly spread channel. This implies that UAC exhibits both delay and Doppler spread. Delay spread leads to time dispersion and frequency selective fading effects. In contrast, time dispersion dilates the transmitted symbol duration in time and needs to be compensated prior to detection. In this special issue, five original technical contributions are addressing problems that will advance the development of next generation underwater communication technologies.

One core high data rate modulation technology that has found its way in modern underwater modem design is Orthogonal Frequency Division Multiplexing (OFDM). OFDM is an efficient method to tackle delay spread induced intersymbol interference (ISI) in underwater acoustic communications. However, a long cyclic prefix is often required and therefore the bandwidth efficiency of the system is reduced. The authors in "Information-theoretic analysis of underwater acoustic OFDM systems in highly dispersive channels" quantitatively analyze the interplay between interference, namely ISI and intercarrier interference (ICI), and maximum transmission rate of the OFDM system. This is accomplished by computing the capacity of OFDM in a doubly spread channel but without relying on the commonly used assumption of the uncorrelated scattering model.

The motion induced time-selectivity of UAC manifests itself as a both frequency or Doppler shift and signal dilation. In multicarrier transmissions employing OFDM, a Doppler shift will introduce ICI to the adjacent subcarriers. Resampling of the received signal will remove both effects, however, leaving residual carrier frequency offsets that need to be compensated. The authors in "Compensation of linear multiscale doppler for OFDM-based underwater acoustic communication systems" investigate the performance of multi-scale Doppler shift compensation in OFDM UAC. It was demonstrated that the proposed algorithms employing coarse timing synchronization can deal with time-varying linear acceleration during a packet made of multiple OFDM frames. In "Performance comparison of Doppler scale estimation methods for underwater acoustic OFDM", the authors compared several Doppler scale estimation methods for zero-padded OFDM systems using a cyclic-prefixed preamble. It is demonstrated that blind approaches exploiting the underlying packet structure work very well at medium to 
high signal to noise ratio ranges, although full or partial knowledge of the transmitted waveform may be required.

The authors in "Underwater localization and tracking of physical systems" address the problem of localization in underwater wireless sensor networks using an OFDMbased physical layer. In many applications, whether civilian or military, the meaningful interpretation of sensed data requires location-aware sensors. This paper presents an underwater localization approach based on one-way message broadcasting from multiple surface nodes. The performance of the proposed solution is evaluated using both simulation and measured results from experiments conducted in a swimming pool and in a local lake using a mobile receiver.

The problem of routing over multihop paths with timevarying link quality is considered in "Location-aware source routing protocol for underwater acoustic networks of AUVs". The authors present the design of a location-aware source routing (LASR) protocol for underwater networks. LASR is based on the sharing of a common time reference between network nodes. Such reference is exploited both for administering multiple access, and for the timely detection of topological changes in the network. LASR is loosely inspired to the Dynamic Source Routing (DSR) protocol. With respect to DSR, LASR employs a different routing metric (cf., the expected number of transmissions before correctly delivering a packet to its destination) and relaxes the source routing approach, by letting the nodes downstream modify the route if topological changes occur. Several mechanisms are employed to improve the effectiveness of multihop communications: these include imposing time constraints over implicit or explicit acknowledging, and performing route maintenance via opportunistic overhearing of neighboring control and data traffic. Simulation results show the good performance of LASR with respect both to a baseline flooding protocol and to DSR, under several environmental and link reliability conditions.

We hope that both academic researchers and engineers working in the area of underwater communications and networking will find the papers presented in this special issue useful and that the contributions and research outcomes in these papers may act as a stepping stone to support future research in this subject.

\author{
Charalampos C. Tsimenidis \\ Yuriy Zakharov \\ Christophe Laot \\ Konstantinos Pelekanakis \\ Paolo Casari \\ Andrey K. Morozov
}



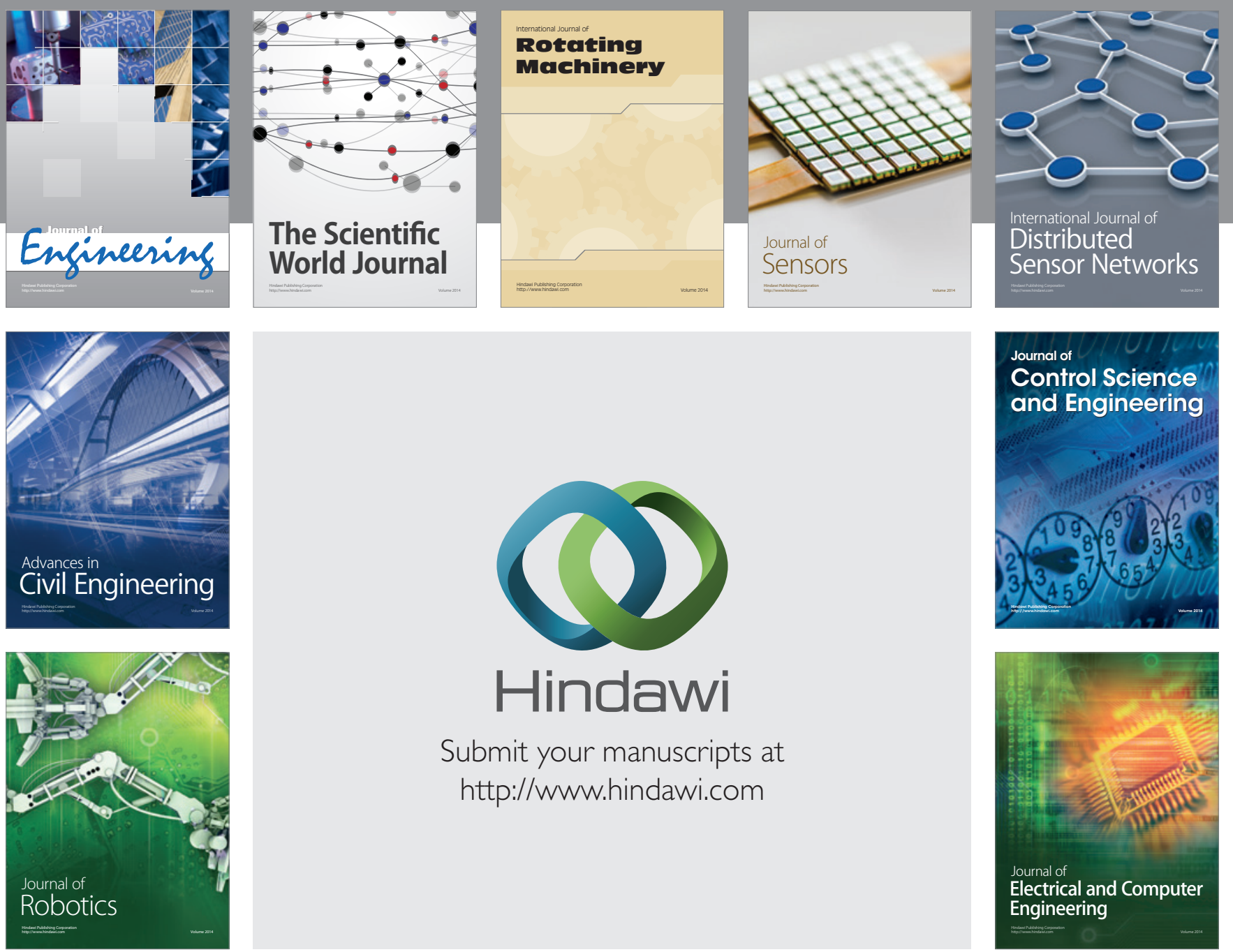

Submit your manuscripts at

http://www.hindawi.com
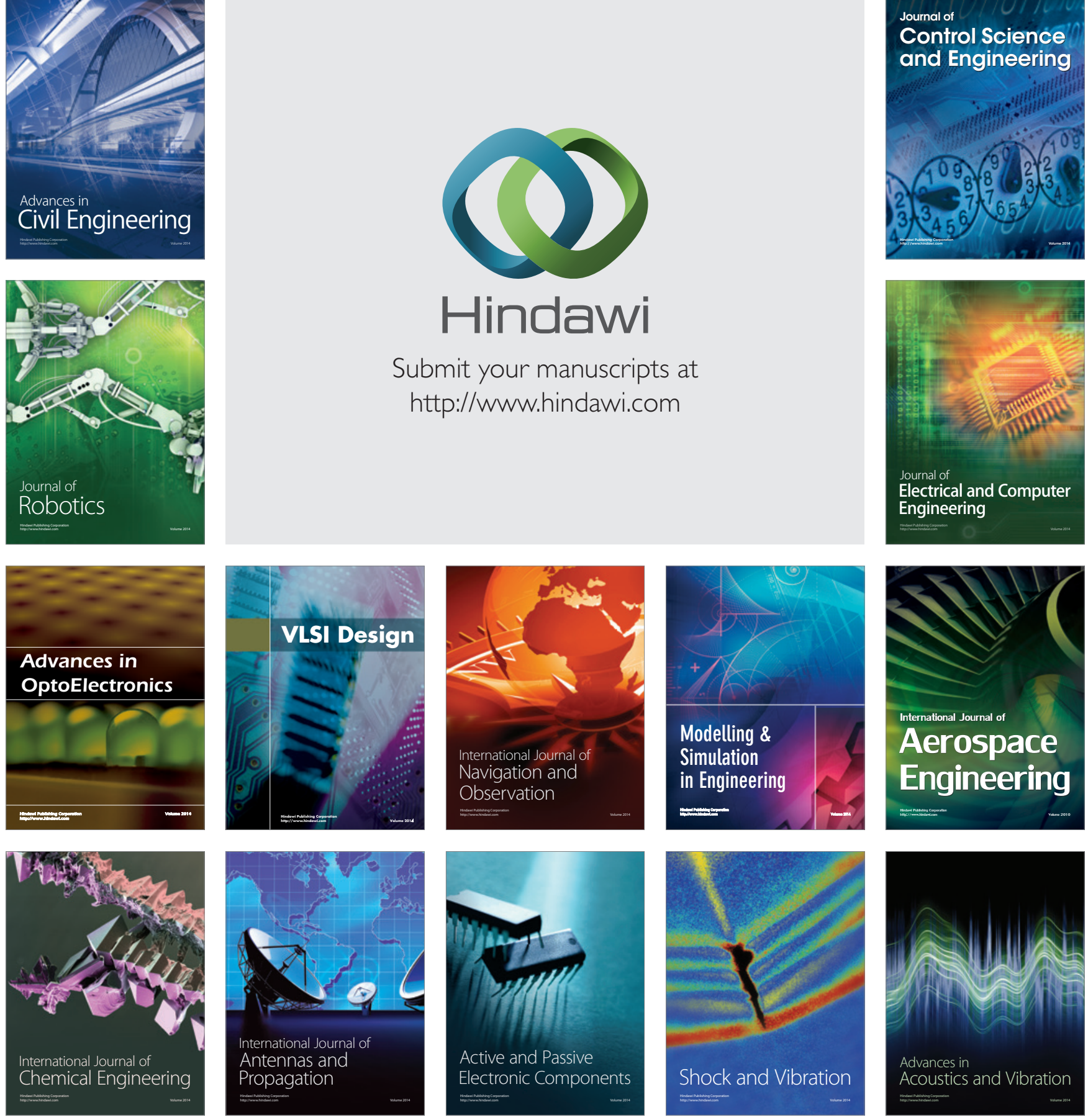